\title{
Bile Acid Synthesis by Long-Term Cultured Cell Line Established from a Human Hepatoblastoma
}

\author{
Yoshiki Amuro, Masanobu Tanaka, Kazuya Higashino, Eijiro Hayashi, \\ Tomoichiro Endo, and Susumu Kishimoto, The Third Department of \\ Internal Medicine, Osaka University School of Medicine, Fukushima-ku, \\ Osaka 553, Japan \\ Hidekazu Nakabayashi and Jiro Sato, Division of Pathology, Cancer Institute, \\ Okayama University School of Medicine, Shikata-cho, Okayama 700, Japan
}

\begin{abstract}
A B S T R A C T Bile acids in the spent medium for the cell culture were analyzed by gas-liquid chromatography and gas-liquid chromatography-mass spectrometry to determine whether human hepatoblastoma cell line could synthesize bile acids. Cholic, chenodeoxycholic, and lithocolic acids were found in the culture medium, and a portion of chenodeoxycholic acid and all of lithocholic acid were sulfated. Since the cells had been cultured in serum-free medium, it is clear that the bile acids were newly synthesized and sulfated by the cultured cells. Chenodeoxycholic acid was the main bile acid in the medium, suggesting that the cell line might predominantly synthesize chenodeoxycholic acid. On the other hand, the cells had fetal or hepatoma characters such as marked $\alpha$-fetoprotein production. These results suggest that fetal or hepatoma type bile acid metabolism might occur in the cell line, and that the established cell line could be an useful in vitro model for the study of bile acid metabolism in hepatoma.
\end{abstract}

\section{INTRODUCTION}

Since bile acids are synthesized from cholesterol in liver, many studies on bile acid metabolism have been performed in liver disease (1). Recently, isolated hepatocyte cultures have been used for studies on the hepatic bile acid metabolism at the cellular levels (24). However, these culture studies were mainly performed using rat-isolated hepatocytes or rat hepatoma. In this study, we analyzed bile acids in the medium used for the cell culture to determine whether the long-term cultured cell line (HUH6-Cl-5), established

Received for publication 29 June 1982. from a human hepatoblastoma by Doi (5), could synthesize bile acids.

\section{METHODS}

Cell culture. The cells have been routinely cultured in serum-free RPMI 1640 medium (Flow Laboratories, Inc., Rockville, MD) supplemented with $3 \times 10^{-8} \mathrm{M}$ sodium selenite. Two batches of spent medium were separately used for bile acid analysis. In our study, the cells were found to produce $\alpha$-fetoprotein markedly $(7,900 \mathrm{ng} / \mathrm{ml}$ culture medium).

Bile acid analysis. All reagents and solvents (Nakarai Chemicals, Kyoto, Japan) for bile acid analysis were of extra grade. Authentic bile acids were obtained from Steraloids Inc., Wilton, $\mathrm{NH} .100 \mathrm{ml}$ of the spent medium, adjusted to $\mathrm{pH} 4$, was applied to a glass column $(2.0 \times 20.0 \mathrm{~cm})$ of $20 \mathrm{~g}$ of Amberlite XAD-2 (Rohm and Haas, Co., Philadelphia, PA). The column was washed with $100 \mathrm{ml}$ of distilled water and eluted with $100 \mathrm{ml}$ of ethanol containing $0.25 \% \mathrm{am}$ monium hydroxide. To separate sulfated bile acids from nonsulfated ones, the eluate was evaporated to dryness and the residue was dissolved in $2 \mathrm{ml}$ of chloroform/methanol ( $\mathrm{i}: 1, \mathrm{vol} / \mathrm{vol}$ ) containing $0.01 \mathrm{M}$ sodium chloride. The bile acid solution was applied to a column of $4 \mathrm{~g}$ Sephadex LH20 (Pharmacia Fine Chemicals, Sweden) prepared in the same solvent (6). Nonsulfated bile acids were eluted with 70 $\mathrm{ml}$ of the solvent. Sulfated bile acids were, thereafter, eluted with $50 \mathrm{ml}$ of methanol and solvolyzed as described (7). The nonsulfated and sulfated bile acids were hydrolyzed (7) and then extracted with ethyl ether after the hydrolyzates were acidified with $2 \mathrm{M}$ hydrochloric acid.

Bile acids were methylated with methanolic 2,2-dimethoxypropane and concentrated hydrochloric acid, and treated with trifluoroacetic acid anhydride. The methyl-trifluoroacetyl (TFA) ${ }^{1}$ derivatives of bile acids were applied to gas-liquid chromatography (Shimadzu model 4CPF, Shimadzu Co., Ltd., Kyoto, Japan) packed with $3 \%$ QF-1 column $(4 \mathrm{~mm} \times 1.5-\mathrm{m}$ glass column$)$. The amounts of bile

\footnotetext{
${ }^{1}$ Abbreviations used in this paper: $\mathrm{RRT}_{\mathrm{D}}$, relative retention time to the methyl-TFA derivative of deoxycholic acid; TFA, trifluoroacetyl.
} 
acids were determined from peak heights on the basis of known amounts of external standards. Bile acids were identified further by gas-liquid chromatography-mass spectrometry (JMS D-300 mass spectrometer, JEOL, Tokyo, Japan). The conditions used were reported previously (7).

\section{RESULTS}

The bile acids were tentatively identified on the basis of relative retention times to the methyl-TFA derivative of deoxycholic acid $\left(\mathrm{RRT}_{\mathrm{D}}\right)$. Fig. 1 shows the gasliquid chromatograms of nonsulfated and sulfated bile acids obtained from the culture medium. The bile acids $\left(R_{R T}\right.$ values of $0.68,1.26$, and 1.93$)$ were analyzed further by gas-liquid chromatography-mass spectrometry. The mass spectra of the bile acids were compared with those of respective authentic bile acids with the same $R_{R T} T_{D}$ values. The methyl-TFA derivative of bile acid with an $R_{R T}$ value of 0.68 had a spectrum with a base peak at mass/energy ratio $(\mathrm{m} / \mathrm{e}) 372[\mathrm{M}-114]$, prominent peaks at $\mathrm{m} / \mathrm{e} 257[\mathrm{M}-(114+115)]$ and $\mathrm{m} / \mathrm{e} 329$ [M - 157], and smaller peaks at $\mathrm{m} / \mathrm{e} 344$ and $\mathrm{m} / \mathrm{e} 486$ [M]; that with an $\mathrm{RRT}_{\mathrm{D}}$ value of $1.26 \mathrm{had}$ a spectrum with a base peak at $\mathrm{m} / \mathrm{e} 369[\mathrm{M}-(114$ $+115)]$, prominent peaks at $\mathrm{m} / \mathrm{e} 370[\mathrm{M}-2 \times 114]$ and $\mathrm{m} / \mathrm{e} 255[\mathrm{M}-(2 \times 114+115)]$, and smaller peaks at $\mathrm{m} / \mathrm{e} 484[\mathrm{M}-114]$ and $\mathrm{m} / \mathrm{e} 598[\mathrm{M}]$; that with an $\mathrm{RRT}_{\mathrm{D}}$ value of $1.93 \mathrm{had}$ a spectrum with a base peak at $\mathrm{m} / \mathrm{e} 154$, prominent peaks at $\mathrm{m} / \mathrm{e} 482[\mathrm{M}-2 \times 114]$ and $\mathrm{m} / \mathrm{e} 367[\mathrm{M}-(2 \times 114+115)]$, and smaller peaks at $\mathrm{m} / \mathrm{e} 253[\mathrm{M}-(3 \times 114+115)]$ and $\mathrm{m} / \mathrm{e} 481[\mathrm{M}$ $-(114+115)]$. These spectra were identical with those of lithocholic, chenodeoxycholic, and cholic acids, respectively. Fig. 2 shows the mass spectra of authentic lithocholic acid and bile acid with an $\mathrm{RRT}_{\mathrm{D}}$ value of 0.68 isolated from the medium.

Quantitative determination of bile acids were performed by gas-liquid chromatographic analysis. The results are shown in Table I. Chenodeoxycholic acid was the main bile acid in both media ( 82.4 and $81.6 \%$ ). All of lithocholic acid was of sulfated form, although $96.1 \%$ of chenodeoxycholic acid and all of cholic acid were of nonsulfated forms. No bile acids were detected in the fresh medium before use.

\section{DISCUSSION}

Although rat hepatocyte and hepatoma cell cultures have been used to study the hepatic bile acid metabolism (2-4), no such metabolic studies have been performed in human hepatoma cultured cells. In this study, we found primary bile acids of humans, cholic and chenodeoxycholic acids, in the culture medium of HUH6-Cl-5 cell line established from a human hepatoblastoma. Since the cells were cultured in serumfree medium and in fact, the fresh medium before

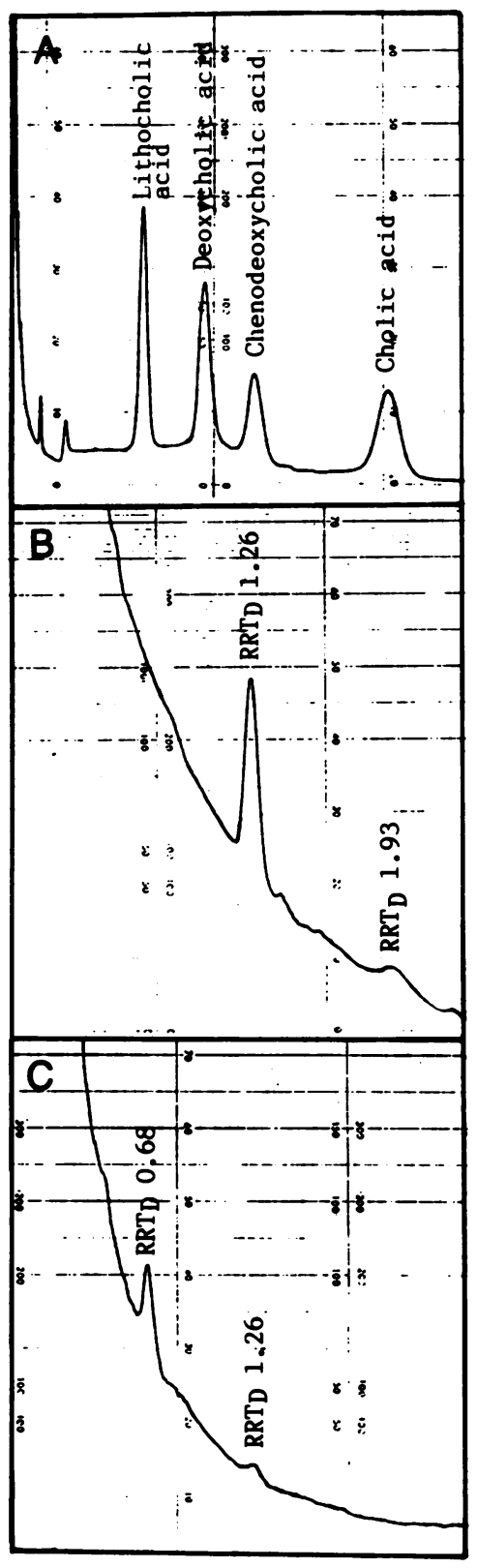

Figure 1 Gas-liquid chromatograms of the methyl-TFA derivatives of authentic bile acids (A), nonsulfated bile acids (B), and sulfated bile acids (C) isolated from culture medium.

culture was free from bile acids, it is clear that these bile acids were newly synthesized by the cultured cells. In the medium, chenodeoxycholic acid concentration was markedly higher than that of cholic acid, suggesting that the cells may synthesize predominantly chenodeoxycholic acid.

Furthermore, we found that lithocholic acid, usually formed from chenodeoxycholic acid by intestinal bac- 


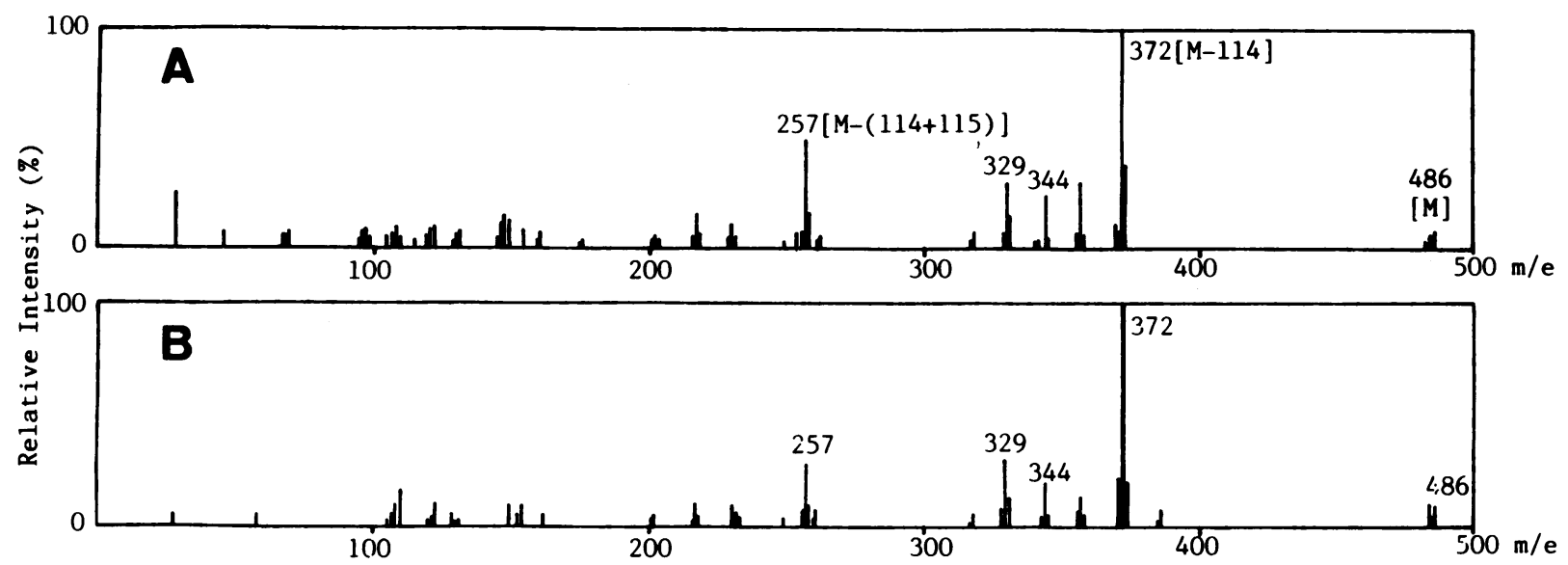

FIGURE 2 Comparison of the mass spectra of the methyl-TFA derivatives of authentic lithocholic acid (A) and bile acid with an $\mathrm{RRT}_{\mathrm{D}}$ value of 0.68 isolated from culture medium (B).

teria in humans, was also present in the medium and all of the acid was of sulfated form. This indicates that the cultured cells are able to synthesize and sulfate lithocholic acid. To our knowledge, this is the first report on lithocholic acid synthesis and sulfation in a cell line derived from human hepatocytes. The presence of lithocholic acid suggests that a portion of chenodeoxycholic acid in the medium might be synthesized from lithocholic acid via an alternative pathway, as proposed by Mitropoulos and Myant (8). Since lithocholic acid, thought to be an intermediate in the pathway, had been found in human meconium (9), amniotic fluid (10), and fetal bile of guinea pig (11), it has been thought that the pathway might operate in mammalian fetal life in the absence of intestinal flora. On the other hand, it is clear that the cell line may have fetal or hepatoma characters, because marked production of $\alpha$-fetoprotein was proven.

In conclusion, the present study suggests that un-

TABLE I

Bile Acids Isolated from Culture Medium

\begin{tabular}{lcc}
\hline & Medium 1 & Medium 2 \\
\hline Total bile acids, $\mu g / 100 \mathrm{ml}$ & 8.5 & 4.9 \\
Lithocholic acid, $\mu g / 100 \mathrm{ml}$ & 1.1 & 0.6 \\
Nonsulfated, \% & 0.0 & $\mathrm{ND}$ \\
Sulfated, \% & 100.0 & ND \\
Chenodeoxycholic acid, $\mu g / 100 \mathrm{ml}$ & 7.0 & 4.0 \\
Nonsulfated, $\%$ & 96.1 & ND \\
Sulfated, \% & 3.9 & ND \\
Cholic acid, $\mu g / 100 ~ m l$ & 0.4 & 0.3 \\
Nonsulfated, $\%$ & 100.0 & ND \\
Sulfated, \% & 0.0 & ND \\
\hline
\end{tabular}

- Not determined. usual, probably fetal or hepatoma type bile acid metabolism might operate in the cultured cells, and that the cell line could be a useful in vitro model for the study of bile acid metabolism in hepatoma.

\section{REFERENCES}

1. Javitt, N. B. 1975. Bile acids and hepatobiliary disease. In Diseases of the Liver. L. Schiff, editor. J. B. Lippincott Company, Philadelphia, PA. 111-145.

2. Anwer, M. S., R. Kroker, and D. Hegner. 1975. Bile acid secretion and synthesis by isolated rat hepatocytes. Biochem. Biophys. Res. Commun. 64: 603-609.

3. Yousef, I. M., J. Ho, and K. N. Jeejeebhoy. 1978. Bile acid synthesis in isolated rat hepatocytes. Can. J. Biochem. 56: 780-783.

4. Lambiotte, $M$. and N. Thierry. 1980. Hydroxylation, sulfation, and conjugation of bile acids in rat hepatoma and hepatocyte cultures under the influence of glucocorticoids. J. Biol. Chem. 255: 11324-11331.

5. Doi, I. 1976. Establishment of a cell line and its clonal sublines from a patient with hepatoblastoma. Gann. 67: 1-10.

6. Makino, I., K. Shinozaki, S. Nakagawa, and K. Mashimo. 1974. Measurement of sulfated and nonsulfated bile acids in human serum and urine. J. Lipid Res. 15: 132138.

7. Amuro, Y., T. Endo, K. Higashino, K. Uchida, and Y. Yamamura. 1981. Urinary and fecal keto bile acids in liver cirrhosis. Clin. Chim. Acta. 114: 137-147.

8. Mitropoulos, K. A., and N. B. Myant. 1967. The formation of lithocholic acid, chenodeoxycholic acid and $\alpha$ - and $\beta$-muricholic acids from cholesterol incubated with rat liver mitochondria. Biochem. J. 103: 472-479.

9. Back, P., and K. Walter. 1980. Developmental pattern of bile acid metabolism as revealed by bile acid analysis of meconium. Gastroenterology. 78: 671-676.

10. Délèze, G., G. Paumgartner, G. Karlaganis, W. Giger, M. Reinhard, and D. Sidiropoulos. 1978. Bile acid pattern in human amniotic fluid. Eur. J. Clin. Invest. 8: 41-45.

11. Li, J. R., and L. Marai. 1977. Sterol and bile acid metabolism during development: 2 . Identification of $3 \beta$ hydroxy-5-cholenoic acid in newborn and fetal guinea pig. Steroids. 30: 815-825. 